\title{
RBD in clinical practice: PSG or questionnaire
}

Keywords: eye movement, parkinson's disease, polysomnography, sleep behaviour disorder, parasomnia

\section{Case report}

Rapid eye movement (REM) sleep behaviour disorder (RBD) is a parasomnia defined by the International Classification of Sleep Disorders (ICSD-3). ${ }^{1}$ It is characterised by abnormal muscle activity during REM sleep, when there normally should be muscle atonia. RBD thus is characterized by REM sleep without atonia (RWA) or intermittent loss of REM sleep atonia on electromyography. Clinically it is characterized by exaggerated motor activity associated with unpleasant and vivid dreams. It may get so violent that it may cause injuries to the patient or the bed partner. Since RBD occurs during REM sleep, it is usually seen after midnight and rarely if ever in the first hour after falling asleep. If a patient is aroused during an RBD episode, he/she can often remember the dream.

RBD is seen in around $0.38-0.5 \%$ of the general population, ${ }^{2,3}$ but is significantly more common in patients with synucleinopathies such as multi-system atrophy (MSA), Parkinson's disease (PD) and diffuse Lewy body dementia (DLD). RBD is observed in up to 15$72 \%$ of unselected PD patients. ${ }^{4,5}$ RBD is therefore considered a strong predictor for development of these disorders. Longitudinal follow up studies suggest that as many as $38-65 \%$ of patients with idiopathic RBD, ${ }^{6,7}$ (some reports as high as $\left.90.9 \%\right)^{8}$ go on to develop a neurodegenerative disease within the next two decades. Thus, diagnosis of RBD carries with it a risk of developing synucleinopathy, which is imminent in near future and that a small window of opportunity for early therapeutic intervention exists.

Diagnosis of RBD is made by clinical history and examination alone, structured questionnaires and polysomnography. The diagnosis of RBD according to ICSD-3 requires the presence of repeated episodes of complex motor behaviours which are documented to occur during REM sleep either by clinical history of dream enactment or polysomnographic correlation. Along with this another mandatory feature required for the diagnosis is demonstration of absence of REM sleep atonia on polysomnographic recording. Thus, the diagnosis of definite RBD today can only be made using polysomnography (PSG). However, PSG even though more sensitive and specific, is time consuming, expensive, not routinely available at most centres and a single nocturnal PSG may not pick up lack of REM sleep atonia and hence will require more than one PSG evaluation.

Eisensehr et al. ${ }^{9}$ compared the efficacy of clinical interview alone without a structured questionnaire to PSG for diagnosing RBD. ${ }^{9}$ This study included 292 patients and showed sensitivity of $33 \%$ and specificity of $90 \%$ for use of clinical interview in PD patients with RBD. On the other hand, another study by De Cock et al reported sensitivity of $87.2 \%$ and a specificity of $90.9 \%$ for clinical interview when compared to video PSG. ${ }^{10}$ It is known that some cases of RBD present with RWA alone and without exaggerated nocturnal movements which can only be picked up on PSG. ${ }^{11}$ This is borne by a study in which $42 \%$ of PD patients had RWA on PSG without any dream enactment behaviours in the overnight PSG. Similar
Volume I Issue 6 - 2017

\author{
Madhuri Behari \\ Department of Neurology, India
}

Correspondence: Madhuri Behari, Professor and Head,

Department of Neurology, India,

Emailmadhuribehari@gmail.com

Received: December 18, 2017 | Published: December 29, 2017

observations were reported by Sixel-Doring, et al. ${ }^{12}$

Low specificity for the clinical methods and questionnaire based study due to the fact that several other disorders present with violent nocturnal behaviours mimicking RBD such as sleep terrors, nocturnal seizures, obstructive sleep apnea (OSA) with agitated REM-related arousals, psychogenic dissociative disorders, and malingering. ${ }^{13}$

To improve the sensitivity and specificity of clinical methods -numerous RBD screening questionnaires have been developed. Two most commonly used questionnaires for screening RBD include REM Behaviour Disorder Screening Questionnaire ${ }^{14}$-RBDSQ (comprising of 10 questions) which has a sensitivity and specificity of around $92 \%$ (though initial reports showed poor sensitivity of around 56\%) and REM sleep behaviour disorder questionnaire-Hong Kong ${ }^{15}$ (RBDQHQ). RBDQ-HK (comprising of 13 questions) has sensitivity and specificity of $87.9 \%$ and $81.3 \%$ respectively. Other questionnaires used for screening include the Innsbruck RBD Inventory (only five

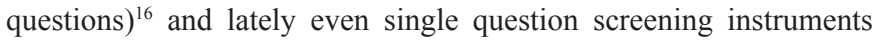
have also been developed. ${ }^{17}$ All these questionnaires have been validated for clinical use and are now in routine use for initial screening for RBD cases. However, even though better than clinical interview alone, with the advantage of easy availability and relative ease of use, they still suffer from the same drawbacks as the clinical interview alone and at best can offer a diagnosis of probable RBD thus making PSG as the only confirmatory technique to diagnose cases of definite RBD.

While, video PSG is the gold standard for diagnosis of RBD as it picks up without any discrimination all cases of RBD without atonia whether or not associated with sleep enactment behaviour, clinical interviews and RBD questionnaires are still useful in routine clinical practice to diagnose RBD with fair degree of confidence save for those patients who lack RBD with atonia or those patients of RBD who do not have abnormal motor behaviour. Head to head trials comparing these two modalities on large population groups and tweaking of the clinical questionnaires to incorporate questions aimed at improving the sensitivity and specificity need to been done so that in the future only a purely clinical diagnosis can be made for RBD.

\section{Acknowledgements}

None. 


\section{Conflicts of interest}

The authors declared that there are no conflicts of interest.

\section{References}

1. Michael JS. International classification of sleep disorders. 3rd ed. Chest Journal. 2014;146(5):1387-1394.

2. Ohayon MM, Caulet M, Priest RG. Violent behavior during sleep. Journal of Clinical Psychiatry. 1997;58(8):369-376.

3. Chiu HFK, Wing YK, Lam LCW, et al. Sleep-related injury in the elderly: an epidemiological study in Hong Kong. Sleep. 2000;23(4):513-517.

4. Gagnon JF, Bédard MA, Fantini ML, et al. REM sleep behavior disorder and REM sleep without atonia in Parkinson's disease. Neurology. 2002;59(4):585-589.

5. Nihei Y, Takahashi K, Koto A, et al. REM sleep behavior disorder in Japanese patients with Parkinson's disease: a multicenter study using the REM sleep behavior disorder screening questionnaire. J Neurol 2012;259(8):1606-1612.

6. Schenck $\mathrm{CH}$, Bundlie SR, Mahowald MW. Delayed emergence of a parkinsonian disorder in $38 \%$ of 29 older men initially diagnosed with idiopathic rapid eye movement sleep behavior disorder. Neurology. 1996;46(2):388-393.

7. Schenck CH, Bundlie SR, Mahowald M. REM sleep behavior disorder: delayed emergence of Parkinsonism and/or dementia in $65 \%$ of older men initially diagnosed with idiopathic REM sleep behavior disorder and an analysis of the minimum and maximum tonic and/or phasic EMG abnormalities found during REM sleep. Sleep. 2003;26(Suppl):A316.

8. Louis SEK, Boeve BF. REM sleep behavior disorder: diagnosis, clinical implications, and future directions. Mayo Clin Proc. 2017;92(11):17231736.
9. Eisensehr I, Lindeiner H, Jager M, et al. REM sleep behavior disorder in sleep-disordered patients with versus without Parkinson's disease: is there a need for polysomnography? J Neurol Sciences. 2001;186(1-2):7-11.

10. Cock DVC, Vidailhet M, Leu S, et al. Restoration of normal motor control in Parkinson's disease during REM sleep. Brain. 2007;130(P2):450-456.

11. Gagnon JF, Bedard MA, Fantini ML, et al. REM sleep behavior disorder and REM sleep without atonia in Parkinson's disease. Neurology. 2002;59(4):585-589.

12. Doring SF, Schweitzer M, Mollenhauer B, et al. Polysomnographic findings, video based sleep analysis and sleep perception in progressive supranuclear palsy. Sleep Med. 2009;10(4):407-415.

13. Schenck CH, Mahowald MW. REM sleep behavior disorder: clinical, developmental and neuroscience perspectives 16 years after its formal identification in SLEEP. Sleep. 2002;25(2):120-138.

14. Kolster SK, Mayer G, Schäfer S, Möller JC, et al. The REM sleep behavior disorder screening questionnaire-a new diagnostic instrument. Mov Disord. 2007;22(16):2386-2393.

15. Li SX, Wing YK, Lam SP, et al. Validation of a new REM sleep behavior disorder question. Sleep Med. 2010;11(1):43-48.

16. Frauscher B, Ehrmann L, Zamarian L, et al Validation of the Innsbruck REM sleep behaviour disorder inventory. Mov Disord. 2012;27(13):16731678.

17. Postuma RB, Arnulf I, Hogl B, et al. A single question screen for rapid eye movement sleep behavior disorder: a multicenter validation study. Mov Disord. 2012;27(7):913-916. 